\title{
Neuronal Ceroid Lipofuscinosis Type 1
}

National Cancer Institute

\section{Source}

National Cancer Institute. Neuronal Ceroid Lipofuscinosis Type 1. NCI Thesaurus. Code C85861.

A condition associated with mutation(s) in the PPT1 gene, encoding palmitoyl-protein thioesterase 1. The condition is one of a group of genetically heterogeneous neurodeg enerative disorders, characterized by accumulation of intracellular lipopigments. 